\title{
El arbitraje en la experiencia latinoamericana
}

\author{
Mario Castillo Freyre* \\ Ricardo Vásquez Kunze ${ }^{* *}$ \\ Rita Sabroso Minaya ${ }^{* * *}$
}

Sumario. 1. Introducción. - 2. La evolución del arbitraje en la década de 1990. - 2.1. Arbitraje de derecho o de conciencia. - 2.2. Arbitraje con el Estado. - 2.3. Arbitraje institucional. - 3. Conclusiones. -

\section{Introducción}

Como se sabe, una de las ideas comunes que aún hoy se tiene sobre Latinoamérica, es la de un continente romántico donde todavía es posible hacer cosas tenidas por vibrantes, emocionantes y épicas que en otras latitudes, como en Europa o en Estados Unidos, es ya imposible hacer.

Nadie se imagina, por ejemplo, a unos guerrilleros encapuchados, anónimos y enigmáticos de ideas, empezar una revolución armada en el bosque de las Ardenas o en la Selva Negra para defender los derechos de los inmigrantes en Europa.

Nadie sueña con que en Francia o en España un Presidente se vista de rojo de la cabeza a los pies y, a punta de plebiscitos, modifique la Constitución de su país cada cierto tiempo, para garantizar que la «revolución» siga

* Magíster y Doctor en Derecho, profesor principal de Obligaciones y Contratos en la Pontificia Universidad Católica del Perú y en la Universidad Femenina del Sagrado Corazón. Catedrático de las mismas materias en la Universidad de Lima, socio del estudio que lleva su nombre <www.castillofreyre.com $>$.

** Abogado del Estudio Mario Castillo Freyre.

*** Profesora adjunta de los cursos de Obligaciones y Contratos Típicos I en la Facultad de Derecho de la Pontificia Universidad Católica del Perú. Alumna de la Maestría en Derecho de la Propiedad Intelectual y de la Competencia en dicha casa de estudios. Miembro del Área de Arbitraje del Estudio Mario Castillo Freyre y Secretaria Arbitral en procesos Ad Hoc. 
su curso y proteja al pueblo del imperialismo malvado que siempre acecha a los gobiernos patrióticos y legítimamente populares.

Nadie se imagina, finalmente, que alguien que no sea un rey, pueda gobernar sin elecciones - $\mathrm{o}$ con elecciones fraudulentas, que es lo mismo- $\mathrm{y}$ de manera absoluta, cuarenta y ocho años una isla, como si no estuviéramos en el siglo XXI sino en el siglo XVI de Enrique VIII.

Pero todo eso que —en otras latitudes - nadie imagina y que por eso parece tan vibrante, como ya hemos dicho, a los espíritus más jóvenes del primer mundo, en Latinoamérica ha sido y hasta hoy sigue siendo posible. De más está decir por eso que las ideas románticas que muchos podrían tener de Latinoamérica son muchas veces ciertas.

El problema de vivir en un continente «romántico» es que, como todo lo romántico, lo exótico o lo novelesco, como ya lo hemos visto con los guerrilleros encapuchados, el presidente vestido de rojo o el seudo rey del poder absoluto en pleno siglo XXI, lo romántico está íntimamente ligado a la locura. Creemos que todos nosotros, independientemente de dónde seamos, hemos sido románticos alguna vez. Y sabemos que ser románticos es perder en cierta forma el juicio. Hacemos cosas que nunca haríamos en nuestros cinco sentidos. $\mathrm{Y}$ por eso, el tercero que hace tratos con un romántico se arriesga a mucho. Para empezar, a que cambie de humores constantemente y que lo que hoy día es «A», mańana sea «B» y pasado mañana «C».

Pues bien, eso es lo que pasa a mayor escala cuando no tratamos ya con una mujer o un hombre romántico, sino con un «Estado romántico»o, peor aún, con toda una comunidad de Estados románticos sumidos en una locura que los lleva a causar una crisis internacional como la de los misiles de 1962, o a exportar médicos para hacer la revolución en otros países, o a nacionalizar los recursos naturales porque se «van a acabar» y se los están llevando como se los llevaban en la Colonia, o — y como sucedió aquí en el Perú hace veinte ańos - no pagar la deuda externa porque no tenemos plata y no nos da la gana, o, finalmente, porque como no me gustó como me miró o me habló tal jefe de Estado en una Cumbre Internacional, anuncio a los cuatro vientos que las empresas del país del jefe de Estado cuyo tonito no me gustó, "tendrán que rendir cuentas». Obviamente, como se comprende, el que hace tratos con semejantes Estados, tiene que someterse a 
las leyes de la inestabilidad emocional que aflige a todos los románticos del mundo. Y lo emocional, de más está decirlo, influirá en lo legal tratándose de un Estado voluble, por lo que el comercio y las inversiones con esos Estados serán siempre de alto riesgo, tanto para sus nacionales como para los extranjeros.

Pero Latinoamérica, gracias a Dios, no es solo ese continente romántico y eterno adolescente con el que muchos inversionistas tienen pesadillas.

Han sido, precisamente, las pesadillas de las que muchos de nuestros países han despertado, las que nos han inmunizado contra el romanticismo político latinoamericano. Economías destruidas peor que en una guerra, sociedades pauperizadas de arriba a abajo donde la pobreza $-\mathrm{y}$ no la riqueza - se ha hecho igual para todos, violencias extremas en nombre de la «justicia social» que han traído más injusticias aun de las que había antes; en fin, todas esas pesadillas han hecho que muchas sociedades latinoamericanas despierten curadas de cualquier romanticismo y emprendan el trabajoso y largo camino de la madurez. Tal esfuerzo es, sin duda, una dura tarea porque mientras la pobreza — que es el mal endémico de nuestros países - siga siendo una realidad, siempre el romanticismo de las revoluciones vengadoras y de los demagogos salvadores tendrán oídos que crean que los discursos maratónicos y los fusiles pueden construir la riqueza y la prosperidad que les falta.

No obstante esto, muchos países latinoamericanos —entre los que se encuentra el Perú, que ha vivido en carne propia las pesadillas de los cuartelazos nacionalistas, de las demagogias electorales y del terror revolucionario- saben perfectamente después de tan amargas experiencias, que la riqueza y la prosperidad no son hijas del romanticismo, sino de la madurez. Y la madurez pasa por los beneficios que puede traer a los pueblos el capital que desarrolla el comercio y la industria en nuestras tierras y que nosotros no tenemos en la cantidad necesaria para derrotar a la pobreza.

Pero los capitales no vienen porque son buena gente. Los capitales vienen a hacer negocios, a obtener ganancias y a que esos negocios y esas ganancias se respeten. Para eso, en primer lugar, se necesita un marco jurídico estable, es decir, todo lo contrario al que ofrece uno de esos Estados románticos, cuyos presidentes cambian las leyes a punta de discursos de acuerdo a la susceptibilidad con la que se despiertan. Y esto requiere a su 
vez una garantía política que no es otra que una garantía institucional. Porque a diferencia de las garantías personales de los jefes de esos Estados románticos que no valen nada (porque siempre valdrá más el «interés nacional», el «interés popular» o «la sagrada causa de la revolución» cuando se les antoje incumplir su palabra), solo los Estados maduros sustentados no en personas sino en instituciones, pueden garantizar el estricto cumplimiento de las leyes que regulan y protegen las inversiones nacionales y extranjeras. Ese es el caso del Perú de hoy.

Pero existe otro hecho importante que hay que resaltar aquí. Además de la estabilidad política y jurídica de un régimen sostenido por instituciones democráticas, el capital requiere de una justicia expedita y eficiente en caso de problemas jurídicos que nunca faltan. Y, lamentablemente, los poderes judiciales no solo de Latinoamérica sino del mundo, no son ya, por razones históricas que no nos proponemos analizar aquí, lo eficientes y expeditos que el capital requiere para su expansión mundial. Por eso es que el capital viene con su propia justicia bajo el brazo. Esa justicia es la justicia arbitral.

\section{La evolución del arbitraje en la década de $\mathbf{1 9 9 0}$}

En este sentido, en América Latina, los mecanismos alternativos de solución de conflictos — tales como el arbitraje y la conciliación — han tenido una evolución notable. A principios de la década de 1990, estos mecanismos eran prácticamente desconocidos. Desde el punto de vista normativo, solo existían anticuadas regulaciones legales sobre arbitraje y sobre conciliación. Además, aquellas regulaciones eran familiares —en exclusiva - para un sector relativamente pequeño de profesionales, además de ser poco estudiadas y escasamente utilizadas.

De esta forma, para fines de la misma década, muchos países de América Latina advirtieron el problema, porque se dieron cuenta de que el capital no venía sin una justicia alternativa a la oficial, lenta y poco fiable a la hora de un conflicto.

En materia de arbitraje, específicamente, el avance latinoamericano en cuanto a la actualización normativa ha sido significativo. Las leyes de arbitraje de la mayoría de los países de América Latina, influenciadas por 
la Ley Modelo de Uncitral, se encuentran a la altura de las leyes más modernas. ${ }^{1}$

Volvemos a señalar que el interés en los mecanismos alternativos de resolución de conflictos en nuestra región, es consecuencia de los profundos cambios económicos y sociales por los que hemos atravesado muchos países que hemos dejado atrás el nefasto romanticismo que ha servido de cuartada para todos los despropósitos políticos, sociales y económicos en América Latina. Ciertamente, la recuperación económica de muchos países que abandonaron las políticas de crear riqueza por decreto o a balazos, causó un repunte en la actividad empresarial, con un subsecuente aumento en las disputas comerciales.

Al mismo tiempo, la democratización que transformó a los sistemas políticos de América Latina durante los últimos años, estimuló la defensa de los derechos individuales, alentando a los ciudadanos a reclamar justicia con más frecuencia y en una mayor variedad de situaciones. En muchos países, la demanda ha sobrecargado la labor de los tribunales ordinarios de justicia que, como ya dijimos, están de por sí en una crisis histórica en todo el mundo.

Los inversionistas extranjeros que llegaron a América Latina - como resultado de los programas de reforma económica y la caída de las barreras comerciales - también han estimulado el interés en los mecanismos alternativos de resolución de disputas. Muy interesados en evitar prolongadas e impredecibles batallas judiciales, estos inversionistas exigen la inclusión de algún tipo de cláusula de conciliación y arbitraje en los contratos que suscriben con gobiernos y empresas latinoamericanas.

Este mecanismo también genera un beneficio indirecto a los ya abrumados sistemas judiciales latinoamericanos, al aliviarles la carga de trabajo.

De esta forma, empezó a producirse un rápido movimiento que se materializó desde dos perspectivas, a saber: por un lado, se dio recepción legislativa a estos instrumentos, con diferentes enfoques; $y$, por otro, se gestaron iniciativas tendientes a su divulgación, también con distintas orientaciones.

CaIvano, Roque J. «Los métodos alternativos de resolución de conflictos en América Latina: logros y desafíos». Revista peruana de análisis, prevención y gestión de conflictos. Año 1, Lima: Centro de Análisis y Resolución de Conflictos de la Pontificia Universidad Católica del Perú, 2007, p. 52. 
En el marco legislativo, algunos países promulgaron leyes directamente orientadas a abarcar estos métodos como, por ejemplo, sucedió en Colombia con el Decreto 1818 de 1998 sobre «Arbitraje y otros procedimientos alternativos de solución de controversias» o en Costa Rica con la Ley 7717 de 1997 sobre «Resolución alterna de conflictos y promoción de la paz social».

Otros países reunieron en una misma ley las normas sobre mediación o conciliación y arbitraje, como sucedió en Bolivia con la «Ley de Arbitraje y Conciliación», Ley 1770 (1997); en Ecuador con la «Ley de Arbitraje y Mediación» (1997); en El Salvador con la «Ley de Mediación, Conciliación y Arbitraje», Decreto Legislativo 914 (2002); en Honduras con la «Ley de Conciliación y Arbitraje», Ley 161 (2000); en Nicaragua con la «Ley de Mediación y Arbitraje», Ley 540 (2005); en Panamá con la «Ley de Arbitraje, Conciliación y Mediación, Decreto Ley 5 (1999); y en Paraguay con la «Ley de Arbitraje y Mediación», Ley 1879 (2002).

Algunos países optaron por regular en cuerpos legales separados los dos principales métodos alternativos, como en el caso argentino en el que se promulgó la «Ley de Mediación para materias civiles y comerciales», Ley 24573 (1995) y la «Ley de conciliación laboral», Ley 24635 (1996), en el ámbito de la justicia nacional, y se dictaron varias leyes de mediación en varias provincias.

Otro ejemplo es el caso peruano, en el que se promulgaron la Ley General de Arbitraje, Ley 26572 (1996) y la Ley de Conciliación, Ley 26872 (1997). Esta regulación en dos normas distintas se sigue manteniendo con el Decreto Legislativo 1071, que regula la nueva Ley de Arbitraje —que entrará en vigencia el 1 de septiembre de 2008 - ${ }^{2}$ y con el Decreto Legislativo 1070, que modifica la Ley de Conciliación.

Algunos países actualizaron su legislación sobre arbitraje. Por ejemplo, encontramos la «Ley de Arbitraje Comercial e Internacional» de Brasil, Ley 9307 de 1996; la «Ley de Arbitraje Comercial e Internacional» de Chile, la Ley 19971 de 2004; la «Ley de Arbitraje Comercial e Internacional» de Guatemala, Decreto 67 de 1995; la Ley modificatoria de las normas sobre arbitraje contenidas en los Códigos de Comercio y de Procedimientos

La Tercera Disposición Final del Decreto Legislativo 1071 «Decreto Legislativo que norma el Arbitraje», establece que «El presente Decreto Legislativo entrará en vigencia el 1 de septiembre de 2008". 
Civiles de 1993 en México; y la «Ley de Arbitraje Comercial e Internacional» de Venezuela, de 1998.

Algunas leyes comprendían tanto el arbitraje nacional como el internacional, pese a que contienen normas diferenciadas para uno y otro, como sucede en Bolivia, Brasil, Ecuador, Perú y Venezuela; mientras que otras normas tratan únicamente el arbitraje comercial internacional, como en el caso chileno.

En conclusión, en la última década y media, la normativa sobre arbitraje ha sido motivo de cambios profundos en Latinoamérica. Durante este lapso, la mayoría de las regulaciones se han reformado, quedando tan solo muy pocos sistemas sin adecuar sus reglas a los modernos principios que los rigen.

Ahora bien, cabe precisar que en la medida de que el derecho aplicable no lo impida u obstaculice, siempre es posible para las partes diseñar los métodos de resolución de conflictos que mejor se adapten a sus necesidades. Para el éxito de dichos métodos, es necesaria una política jurídica orientada hacia la resolución extrajudicial de los conflictos, un cuerpo profesional de abogados entrenados en el uso de dichos métodos y una judicatura dispuesta a colaborar con estos mecanismos extrajudiciales, prestando la cooperación judicial necesaria para que los diversos métodos cuenten con la confianza de sus usuarios.

\subsection{Arbitraje de Derecho o de CONCIENCIA}

Cabe destacar que la reciente ola de reformas a la legislación de arbitraje en América Latina ha preservado la distinción entre arbitraje de derecho y de equidad. Algunas legislaciones, como la Ley General de Arbitraje, Ley $26572,{ }^{3}$ han llegado a mantener el principio que establece que el arbitraje debe ser considerado de equidad, a menos que las partes se hayan pronunciado expresamente a favor del arbitraje de derecho.

\footnotetext{
El artículo 3 de la Ley General de Arbitraje, Ley 26572, establece lo siguiente:

Artículo 3.- «Arbitraje de derecho o de conciencia.-

[...]

Salvo que las partes hayan pactado expresamente que el arbitraje será de derecho, el arbitraje se entenderá de conciencia.

$[\ldots] »$.
} 
$\mathrm{Al}$ respecto, Alejandro Garro ${ }^{4}$ señala que la distinción conceptual entre arbitraje de derecho o de conciencia se encuentra muy enraizada en aquellas jurisdicciones en donde se ha venido aplicando durante mucho tiempo. Esta es la situación en que se encuentran los países latinoamericanos, donde el arbitraje de jure y ex aequo et bono cuenta con un reconocido linaje.

La distinción en el arbitraje de derecho y la amigable composición ha sido utilizada para trazar líneas divisorias en donde era innecesario o poco conveniente trazarlas. Por lo general, la distinción ha sido útil, a efectos de expresar la voluntad de las partes de otorgar a los árbitros amplias facultades para decidir el conflicto, independientemente de las reglas de derecho que le fueren aplicables, para distinguirlo de aquel arbitraje en el cual los árbitros solo pueden dictar un laudo conforme a las normas de derecho aplicables.

Fundamentalmente por este motivo, y no por otro, es que Garro es partidario de mantener la distinción, que por lo visto ha contado con la comprensión y aceptación de la mayoría de la doctrina y de los usuarios en América Latina, en donde la amigable composición cuenta con sus seguidores.

$\mathrm{Al}$ respecto, debemos resaltar que nuestra nueva Ley de Arbitraje - a diferencia del artículo 3 de la Ley General de Arbitraje, Ley 26572 — ya no contempla un artículo que regule expresamente el tema de la presunción sobre la clase de arbitraje (de "conciencia» o de «derecho»), en caso las partes no lo hayan pactado.

Sin embargo, el inciso 3 del artículo 57 de la nueva Ley de Arbitraje —que regula las «normas aplicables al fondo de la controversia»— establece que "[...] el tribunal arbitral decidirá en equidad o en conciencia, solo si las partes le han autorizado expresamente para ello" (El subrayado es nuestro).

Como se puede apreciar, ahora se presumiría iuris tantum que se trata de un arbitraje de derecho, es decir, que los árbitros deben resolver la cuestión controvertida con arreglo al derecho aplicable, salvo que las partes hayan establecido que se trata de un arbitraje de conciencia (también denominado como de equidad en la nueva Ley).

4 Rubio GuerRero, Roger. «Globalidad, integración y arbitraje (entrevista a Alejandro M. Garro)». Themis, $\mathrm{n}^{\circ}$ 42. Revista editada por alumnos de la Facultad de Derecho de la Pontificia Universidad Católica del Perú, Lima, 2001, p. 216. 


\subsection{Arbitraje con el Estado}

Por otro lado, debemos reiterar que los inversionistas extranjeros que llegaron a América Latina han estimulado el uso de los mecanismos alternativos de resolución de conflictos, ya que exigían la inclusión de algún tipo de cláusula de conciliación y arbitraje en los contratos que suscribían con, por ejemplo, los Estados.

Sin embargo, por tradición, Latinoamérica, apegada profundamente al concepto de soberanía del Estado, ha visto grandes inconvenientes en que este o los entes que forman parte de él, se sometan a arbitraje.

$\mathrm{Al}$ respecto, Bernal Gutiérrez ${ }^{5}$ nos dice que esta circunstancia se ha hecho evidente en dos frentes: el interno, donde la reticencia y resistencia — movida por intereses de diversa índole — ha sido férrea y sostenida; y el externo, donde, a pesar de estar casi sin excepción vinculados y adheridos a los tratados más importantes, se desconocen estos mecanismos en nombre de la soberanía y la preponderancia de la Constitución, como consecuencia del fanatismo e ignorancia.

En este ambiente no era raro encontrar, como hace una década, que la normativa latinoamericana vigente en materia de solución de controversias, donde fuere parte integrante el Estado o sus entes y, en particular, la referida a la solución de controversias contractuales, no registrara en términos generales ninguna disposición que tratara explícitamente el tema.

No quiere ello decir que no se llevaran a cabo arbitrajes, ni que la mecánica no se utilizara o que no fuere considerada como una buena opción. Simplemente, la tendencia imperante era la de favorecer al Estado y generar para él — tan solo por ostentar tal condición- escenarios de privilegio, sin importar que el Estado se encontraba en el mismo nivel que los inversionistas y que ello no comprometía —en modo alguno- la soberanía ni los poderes del Estado. Se trataba del Estado o sus entes y, por ende, tenía derecho a tal privilegio, sin importar nada más y sin consideración alguna.

5 Bernal GutiérReZ, Rafael. «El arbitraje del Estado: la regulación en Latinoamérica». Revista Internacional de Arbitraje. Bogotá: Legis, junio-diciembre 2004, pp. 123-124. 
Como bien señala Bernal, no obstante, quizás por la influencia de los compromisos adquiridos a escala internacional y por la necesidad de encontrar una mayor y mejor dinámica interna, los inconvenientes a los que se ha hecho referencia han cedido de manera relativa. Hoy en día, como consecuencia de la reforma que venimos comentando, podemos observar una referencia constante a la materia, permisiva en líneas generales, de la aplicación del arbitraje y de los métodos alternativos de resolución de controversias, cuando del Estado y sus entes se trata. Esto, sin desmedro de sus poderes, pero quizá con un tono menor y un más claro entendimiento del concepto de «soberanía» y con mayor conciencia del papel que desempeñan cuando, como particulares, contratan o causan daños.

Para respaldar este punto de vista, demos una mirada panorámica a las normas vigentes en materia de arbitraje en Latinoamérica. Así, podemos distinguir dos bloques; a saber: aquellos países que no han contemplado — con relación al sometimiento del Estado a arbitraje — ninguna regulación; y aquellos otros que sí lo han hecho ${ }^{6}$.

En el primer bloque, encontramos, por ejemplo, a Argentina y Uruguay, en donde sus ordenamientos internos no contemplan una norma concreta sobre el sometimiento del Estado al arbitraje. Sin embargo, ello no ha impedido, por un lado, que dichos países se hayan integrado a diversos tratados donde tanto los particulares como el Estado se encuentran sometidos a resolver sus controversias por la vía arbitral, sino que, de otro lado y en el campo interno, el arbitraje se practica y es utilizado sin reticencias y sin que para la doctrina haya sido objeto de cuestionamientos o dudas que hagan del arbitraje una herramienta desechada para el caso.

Incluso, en el plano internacional, Argentina y Uruguay no solo son signatarias y adherentes de las convenciones de Nueva York, Panamá y Montevideo, sino que, como partes integrantes del Mercado Común del Sur (Mercosur), han suscrito y ratificado todos los instrumentos que le han dado vida, en particular, los que han tenido por objeto regular lo referente a la solución de controversias, bien entre los Estados partes o bien entre estos y los particulares (inversionistas) o los particulares de estos Estados entre sí.

6 Bernal GutiérRez, Rafael. Ob. cit., pp. 124-143. 
En este ámbito cabe destacar el «Protocolo de Brasilia» y su Reglamento, igualmente aplicables, por extensión, a Brasil, Bolivia y Chile debido a su adhesión al Mercosur.

Otro ejemplo de este primer bloque lo ofrece México. Sin embargo, el régimen de arbitraje mexicano es fiel a la Ley Modelo de UnCitral y respecto de las materias tratadas, la Ley Modelo es trascripción de lo dispuesto casi integralmente por la Convención de Nueva York, por lo que se ha afirmado — sin reticencia alguna - que resulta aplicable tanto a entres privados como a públicos. Ello, en la medida de que en todas estas disposiciones no se hace distinción o discriminación de ninguna clase, ni tampoco se contempla prohibiciones al respecto. De esta manera, tanto Estado mexicano como sus entidades pueden someter sus diferencias al arbitraje lo que, de hecho, ocurre de manera frecuente.

En el plano internacional, no podemos dejar de hacer referencia al Tratado de Libre Comercio de México con Estados Unidos y Canadá (TLCAN), donde la mecánica arbitral está contemplada tanto para las diferencias entre los Estados signatarios, como entre ellos y los particulares (inversionistas) y, a su vez, entre los mismos particulares de dichos Estados.

En consecuencia, al igual que ocurre en Argentina y México, la firma de un número cada vez más creciente de tratados bilaterales de inversión ha traído consigo la obligatoriedad del arbitraje sin distinción alguna, con lo cual se ha reafirmado de manera sólida la utilización del mismo para la solución de controversias entre partes, incluidos el Estado y sus entes.

Ahora bien, entre los países que sí contienen normas particulares sobre la participación del Estado en el arbitraje o en algún medio alternativo de solución de conflictos, encontramos a Guatemala, Costa Rica, Honduras, El Salvador, Ecuador, Bolivia y Paraguay.

El Perú cuenta —en opinión mayoritaria— con una completa legislación sobre la materia. En efecto, el artículo 2 de la Ley General de Arbitraje, Ley 26572, contempla clara y enfáticamente la posibilidad de acceder al arbitraje por parte del Estado, al señalar que «pueden ser sometidos a arbitraje nacional, sin necesidad de autorización previa, las controversias derivadas de los contratos que celebren el Estado Peruano y las personas jurídicas de derecho público con nacionales o extranjeros domiciliados en el país, inclusive las que se refieran a sus bienes, así como aquellas 
controversias derivadas de contratos celebrados entre personas jurídicas de derecho público, entre sí».

Asimismo, el inciso 2 del artículo 4 de la nueva Ley de Arbitraje, Decreto Legislativo 1071 (que entrará en vigencia el 1 de septiembre del presente año), establece que «las controversias derivadas de los contratos y convenios celebrados entre estas entidades estatales pueden someterse también a arbitraje nacional», mientras que el inciso 3 señala claramente que «el Estado puede someter a arbitraje nacional las controversias derivadas de los contratos que celebre con nacionales o extranjeros no domiciliados en el país».

Por su parte, el artículo 92 de la Ley General de Arbitraje, Ley 26572, establece que «Pueden ser sometidas a arbitraje internacional dentro o fuera del país, libremente y sin requisito de previa autorización, las controversias derivadas de los contratos que el Estado Peruano y las personas de derecho público celebren con nacionales o extranjeros no domiciliados, así como las que refieren a sus bienes», criterio que se mantiene en el inciso 4 del artículo 4 de la nueva Ley de Arbitraje.

En consecuencia, como se ha podido apreciar, en el movimiento de reforma y actualización legislativa que se ha producido en Latinoamérica existe un franco ánimo de involucrar al Estado y los entes públicos en la utilización del arbitraje.

A pesar de que no existe unanimidad y ni siquiera uniformidad en el tratamiento, se destacan algunos países proclives al efecto y otros en donde con restricciones es aceptado.

Representa un anhelo innegable que, a partir de la nueva estructura legislativa, se logre consolidar y extender el arbitraje, a fin de que se imponga como la herramienta que a todos conviene y, en particular, a los Estados y a los inversionistas.

\subsection{Arbitraje InSTITUCIONAL}

Finalmente, nos corresponde abordar el auge y la expansión del arbitraje institucional en Latinoamérica, habida cuenta de que es innegable que el desarrollo explosivo del arbitraje institucional constituye un fenómeno en aumento, típico de la última década del siglo pasado. 
Así, por ejemplo, Berizonce ${ }^{7}$ señala que en Brasil, la Ley de Arbitraje de 1996, no obstante la inexistencia de una cultura favorable, generó una multiplicación de los órganos arbitrales institucionales. No solo se modernizaron los existentes sino que se crearon nuevos, especialmente en los grandes centros urbanos, multisectoriales, con el apoyo de las asociaciones mercantiles.

La misma tendencia se advierte en la Ley de Arbitraje Comercial de Venezuela de 1998, que regula minuciosamente el «arbitraje institucional», previendo que una amplia gama de entidades no gubernamentales están autorizadas para organizar sus propios centros de arbitraje, dictando los respectivos reglamentos.

No han sido ajenas a esta línea las leyes de arbitraje de Colombia, Costa Rica, Uruguay, México, entre otros países.

Cabe señalar que a partir de los años noventa, se han conformado órganos de arbitraje permanentes en el seno de los colegios profesionales de abogados, notarios y de ingenieros. Así, solo por citar algunos ejemplos locales, tenemos el Centro de Arbitraje del Colegio de Abogados de Lima, el Centro de Arbitraje del Colegio de Ingenieros del Perú (en sus distintas sedes departamentales) y el futuro Centro de Arbitraje del Colegio de Notarios de Lima.

Latinoamérica, en definitiva, asiste en las dos últimas décadas al remozamiento del arbitraje tradicional, a partir de la sanción de leyes especiales o a la actualización de los ordenamientos procesales para dar cabida, entre otras modalidades, al arbitraje institucional.

\section{Conclusiones}

Para concluir, solo nos queda señalar que las normas no lo son todo si esa mentalidad adolescente y romántica de querer cambiarlo todo de la noche a la mañana y que ha sido, como hemos señalado al principio, la gran pesadilla de Latinoamérica, no cambia también.

BerIzONCE, Roberto Omar. «El arbitraje institucional en Iberoamérica». Derecho PUC, N 53, Lima: Pontificia Universidad Católica del Perú, 2000, pp. 759-760. 
Para eso, el capital, el comercio y las inversiones tienen que ser el sustento de una nueva forma de pensar, porque sin esa nueva forma de pensar de nada valdrán las más modernas leyes. Esa nueva forma de pensar, que es que el capital no trae explotación sino prosperidad y desarrollo, es una tarea compartida. Una tarea de los inversionistas y de su compromiso de ganar generando oportunidades reales de progreso a los latinoamericanos, sobre todo a los que menos tienen; y una tarea de los propios pueblos, aprovechando esas oportunidades de desarrollo y siendo justos con quienes nos las dan. Creemos que así dejaremos de una vez las utopías románticas que tanto han marcado nuestra historia. 